\title{
Dialectical behaviour therapy for borderline personality disorder
}

\author{
Robert L. Palmer
}

This is the final in a series of papers in APT devoted to innovative cognitive psychotherapies. The previous papers discussed cognitive-analytic therapy (Denman, 2001), very brief dynamic psychotherapy (Aveline, 2001) and problem-solving therapy (Mynors-Wallis, 2001).

The concept of borderline personality disorder (BPD) seems to include, almost by definition, the idea that people described by the term are difficult to help. The broad picture portrayed by the diagnostic criteria (Box 1) is likely to be familiar to most clinicians and to cause the hearts of many to sink. A core issue for those with BPD is difficulty of relationship, and this will inevitably - even especially - include clinical and therapeutic relationship. It is all too common for distress to be met with much therapeutic effort but for little to change. Indeed, there is often a nagging sense that attempts at treatment may be making matters worse. The care of people with BPD presents an important challenge to mental health services. At the individual level, the patient continues to be at risk and to suffer and the clinician feels frustrated. At the service level, substantial resources may be expended to little apparent benefit.

\section{Box 1 DSM-IV diagnostic criteria for borderline personality disorder}

A pervasive pattern of instability of interpersonal relationships, self-image, and affects, and marked impulsivity beginning by early adulthood and present in a variety of contexts, as indicated by five (or more) of the following:

(1) frantic efforts to avoid real or imagined abandonment. Note: Do not include suicidal or selfmutilating behavior covered in Criterion 5

(2) a pattern of unstable and intense interpersonal relationships characterized by alternating between extremes of idealization and devaluation

(3) identity disturbance: markedly and persistently unstable self-image or sense of self

(4) impulsivity in at least two areas that are potentially self-damaging (e.g., spending, sex, substance abuse, reckless driving, binge eating). Note: Do not include suicidal or self-mutilating behaviour covered in Criterion 5

(5) recurrent suicidal behavior, gestures, or threats, or self-mutilating behavior

(6) affective instability due to a marked reactivity of mood (e.g., intense episodic dysphoria, irritability, or anxiety usually lasting a few hours and only rarely more than a few days)

(7) chronic feelings of emptiness

(8) inappropriate, intense anger or difficulty controlling anger (e.g., frequent displays of temper, constant anger, recurrent physical fights)

(9) transient, stress-related paranoid ideation or severe dissociative symptoms

(American Psychiatric Association, 1994: p. 654)

Bob Palmer is the Clinical Director of the Leicestershire Eating Disorders Service and an honoray consultant psychiatrist and senior lecturer at Leicester General Hospital (University Department of Psychiatry, Brandon Mental Health Unit, Leicester General Hospital, Leicester LE5 4PW, UK). He is the author of Helping People with Eating Disorders and other books and papers on the topic of the clinical eating disorders. 
If the worried clinician or service manager turns to the literature, there are many opinions on offer about how people with BPD may best be helped but much less in the way of clear evidence (Kisely, 1999). To date, there have been few randomised controlled trials (Perry et al, 1999). Dialectical behaviour therapy (DBT) is unusual in having been evaluated in two such trials. The first showed that DBT was superior to community-based 'treatment as usual' in women with BPD associated with repeated self-harm (Linehan et al, 1991). Some benefit was retained at follow-up (Linehan et al, 1993, 1994). A further study showed similar benefits in women with BPD and substance misuse (Linehan et al, 1999). Thus, DBT can claim an evidence base of a kind that other psychotherapeutic treatments currently lack. For this reason, it has been increasingly used in the USA, where it has been taken up with great enthusiasm. Sometimes, its advance has been associated with a degree of brouhaha that can be a little off-putting. Now DBT has arrived in the UK. What place should it have in British practice?

\section{The elements of DBT}

Dialectical behaviour therapy is the brainchild of Marsha Linehan, a clinical psychologist from Seattle with an impressive academic track record. She is the author of a book describing the treatment and of an accompanying skills training manual (Linehan, $1993 a, b)$ However, although Linehan is the creator and undoubted guru of the enterprise, many others have contributed and the development of the treatment continues. The explicit aim has been and remains to create a practical way of helping people who are otherwise very difficult to treat. In tune with this pragmatic stance, DBT is a hybrid created out of a variety of disparate elements. It seems to have a number of intellectual roots. Some are in its unusual name. Some are not. And at first sight, they form a strange collection.

\section{Cognitive-behavioural therapy}

One of DBT's several elements is cognitivebehavioural therapy (CBT). DBT espouses the scientific ethos. It makes use of self-monitoring, there is an emphasis on the here and now and much of the therapeutic technique is borrowed from CBT, including the style of open and explicit collaboration between patient and therapist. Furthermore, the treatment has a manual. The 'bible' of DBT is Linehan's Cognitive Behavior Treatment of Borderline Personality Disorder (1993a). (She says that her publisher insisted on 'cognitive behavior treatment' because they felt that a title including the word dialectical was less likely to sell well, particularly in the USA.)

\section{Dialectical thinking}

The 'dialectical' in DBT refers to the way in which it uses a broad way of thinking that emphasises the limitations of linear ideas about causation. It substitutes 'both/and' for 'either/or' and sees truth as an evolving product of the opposition of different views. The use of dialectical ideas in DBT arises largely from the clinical observation of the mixed and shifting nature of human emotion and experience in general and in patients with BPD in particular. Interaction with such a patient is unlikely to have the characteristics of a logical argument or even a orderly conversation. It is more likely to be akin to a dance to rapidly changing music in which clinician and patient each react to the other sometimes to good effect, sometimes with much painful stamping on toes.

\section{A touch of Zen}

Dialectical thought emphasises the wholeness and interconnectedness of the world and the potential for the reconciliation of opposites. This emphasis is shared by the third element in DBT: the use of thinking and techniques drawn from Zen Buddhism. The key concept is that of mindfulness. The person with BPD is seen as having especial difficulties in being at all detached from his or her experience and, indeed, as often being overwhelmed by it. Developing the capacity for being mindful and living in the moment allows a greater potential for feeling appropriately in charge of the self. Zen is full of paradox, and there is something paradoxical about seeking greater mastery through a kind of detachment. However, when the alternative is being engulfed, then the place of mindfulness becomes clear. A related concept is the balance between acceptance and change. The most difficult idea for some is that the world is as it is. But again there is some paradox in the notion that acceptance - for instance, of unchangeable traumatic events in the past - may be necessary for change to be possible.

\section{Speaking metaphorically}

The metaphor of dance was employed above in discussing dialectics, and it is used quite often as a model of the therapeutic relationship in DBT. Indeed, DBT is bedecked with metaphors. Along with its 
apparently esoteric elements, it is characteristic of DBT that it also aspires to be down-to-earth. It contains a large measure of common sense expressed in folksy language, sometimes in a slightly selfconscious way. Aphorisms abound both in the handbook and in the hand-outs for patients. For example, the overriding attitude of the therapist should be that "patients are always doing the best they can" and that it is necessary to find the "kernel of truth" in the thoughts and assumptions of the sufferer. The need to try to gain something out of a set back or even a disaster is described as "making lemonade out of lemons". The active nature of the process from the patients' point of view is expressed by the saying that patients "may not have caused all of their own problems but they have to solve them anyway". Being effective is more important than being right. There is talk of discovering one's "wise mind". In general, therapy is about keeping things on the move. Furthermore, the judicious use of humour - "irreverence" - is advocated as a useful technique for keeping the therapeutic dance on the go when it might otherwise slow to a halt. Training may contain the slightly surreal experience of would-be therapists being asked to share their best funny lines.

\section{The biosocial theory}

Dialectical behaviour therapy is thus a strange hybrid. Things that simply seem to work have been included. It is not essentially theory driven, and what theory there is is limited. Its working model is the biosocial theory of BPD (Fig. 1).

The biosocial theory suggests that BPD is a disorder of self-regulation, and particularly of emotional regulation, which "results from biological irregularities combined with certain dysfunctional environments, as well as from their interaction and transaction over time" (Linehan, 1993a: p. 42).

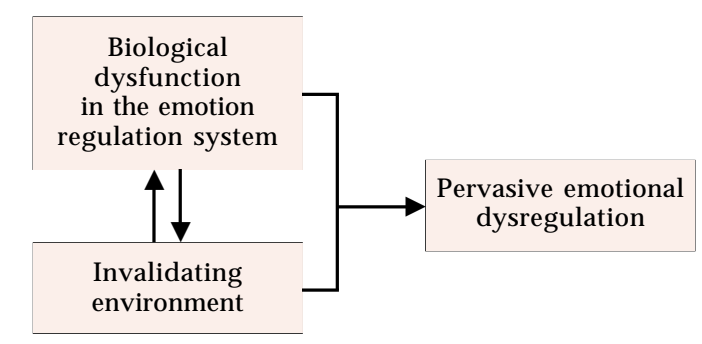

Fig. 1 Biosocial theory of borderline personality disorder
People with BPD are seen as being unusually emotional by temperament but as having had this trait rendered maladaptive by a serially invalidating environment. They may have been made to feel that their emotional reactions are unjustified, inappropriate or a sign of illness. Vicious circles develop and the individual becomes trapped in the selfperpetuating cycle of BPD. Self-invalidation may make an important contribution once the process is underway and, of course, the individual's reactions may provoke undermining reactions from others. Linehan advocates the biosocial theory as one way of beginning to understand BPD and as likely to "engender an attitude of effective compassion" in clinicians. She sees individuals with BPD as being "involuntarily in hell", and the role of DBT is to help them to get out and stay out. The theory is explained to the patient as part of the process of commitment to treatment.

\section{Components of DBT}

Dialectical behaviour therapy is a treatment with several components. In its usual form it is an outpatient treatment delivered over a period of 1 year, although patients may join a DBT programme for a second year or stay in therapy for even longer. The main components for the patients are a weekly individual out-patient session, attendance at a weekly skills training group and possible out-ofhours telephone contact. In addition, for the therapy team there is a weekly consultation meeting. Other treatment interventions such as drug treatment or emergency crisis admission may also occur, but these are not essential components of DBT .

\section{Individual sessions}

Individual sessions typically last for 45-60 minutes and occur weekly. The past week is reviewed using 'diary cards' that the patient has filled in. There is a recommended structure to sessions that follows a hierarchy of topics. Life-threatening behaviours should be discussed and dealt with first. Then come therapy-interfering behaviours, quality-of-lifeinterfering behaviours and attention to skills. In practice, the detailed content of sessions may be influenced by the style of the individual therapist. However, the whole process should be aimed at providing a setting that is validating for the patient. Typically, a good deal of attention is paid to the immediate antecedents of troublesome feelings and actions, and detailed chain analysis may be used to work out exactly how a particular crisis came about. 
There are forms that may help the patient and therapist to map out apparent chains of causation. At least at first, the emphasis is on the promotion of the greater use of skills to survive and manage difficult feelings. These skills are taught mainly within the weekly training group.

\section{Skills training group}

Skills training is conducted in a weekly group, which typically lasts for 2 or $2 \frac{1}{2}$ hours. The style of the group is didactic. Therefore the room may be arranged like a classroom, with the skills trainers usually two - sitting at the front. The skills trainers may be also the individual therapists of some of the group members but this dual relationship is not attended to within the group. Process issues and evident emotion in the group are discussed and dealt with only if they are life-threatening or, more commonly, if they are noticeably interfering with therapy. Thus, that someone appeared sullen and inattentive would be addressed only if his or her behaviour was causing a major disruption to the running of the group. It would not be addressed as an issue in its own right or as an illustration of wider problems. This sometimes leads the trainers to have to carry on regardless of a group that seems to have its attention elsewhere. However, appearances may be deceptive. Or so the therapist has to hope.

The skills training is organised around a manual that sets out the content of the programme in detail and gives advice about how it should be taught (Linehan, 1993b). The book also contains hand-outs for patients, which may be freely copied for the purpose. Groups may include group exercises and role-play, and homework is suggested.

The content is divided into four modules, each of which is usually taught in blocks lasting a few weeks. Three modules are on topics - emotional regulation, distress tolerance and interpersonal effectiveness - similar to those found in other CBT and related programmes. The other module is on mindfulness. The mindfulness module is often repeated in short form between each of the other modules. Furthermore, it is typical for every skills training group to begin with a mindfulness exercise.

\section{Out-of-hours telephone contact}

Patients within a DBT programme may telephone their individual therapist between sessions. Pagers are often used for the initial contact. Such contact may sometimes be planned but is chiefly about helping the patient to get through crises. The contact should be mainly about coaching the patient in the appropriate use of the skills that have been learned previously in the group. Typically a DBT telephone call is brief - perhaps 5 or 10 minutes - and should not in general be used as an extra therapy session. Its function is different. It is mainly to try to help the patient avoid self-harm or sometimes to sustain or repair the therapeutic relationship. The patient is banned from contacting the therapist for 24 hours after an act of self-harm.

The hours during which telephone contact is available are agreed between the therapist and the patient. What the therapist can manage is an explicit determinant and, indeed, is usually the limiting factor. The arrangement may be changed if it is misused. However, perhaps surprisingly, even highly disturbed patients usually respect the rules of the game. If they do not, this is discussed exhaustively as therapy-interfering behaviour.

\section{The consultation group}

The fourth essential component of DBT does not involve the patient directly. This is the weekly consultation group. DBT is a team treatment and the consultation group is where the team - the individual therapists and skills trainers - meet to review the programme and their practice. The dialectical style that characterises the practice of DBT with patients is continued even within this peer supervision group. The group is there "to treat the therapist", pushing and pulling appropriately to keep the process of therapy on the move.

\section{Commitment}

Before DBT can begin, the patients have to commit themselves to participation in the therapy. This is an exercise in itself and may take several meetings. Both the patient and the therapist make explicit commitments (Box 2). In practice, the therapist may initially 'play hard to get' and lead the patient, to persuade him or her that the programme is indeed justified. People with BPD have not uncommonly had experiences of previous treatments that have been at best unrewarding. Consequent wariness needs to be validated and the new therapeutic endeavour presented in a realistic way as promising but also demanding. Time spent on commitment before therapy is a good investment. Likewise, if the therapeutic relationship becomes wobbly or threatens to break down, then time needs to be spent on explicit recommitment. It is usual for there to be an agreement that if three consecutive sessions of one kind are missed for any reason then the patient is out of the DBT programme. 


\section{Consultant to the patient}

Once the patient is in the programme the usual stance of the therapist is that of being a consultant to the patient. Whenever possible the patient is left as the agent of his or her life, with the therapist available for support, advice, coaching and 'cheerleading'. Sometimes this may run counter to usual clinical practice and may consequently present the therapist with dilemmas. For instance, the classic DBT stance with patients who find themselves in a crisis in an accident and emergency (A\&E) department would be not to advise the A\&E clinicians on how to manage the patient but to advise the patient on how to manage the A\&E staff. However, this stance is not absolute and DBT is characteristically pragmatic. The patient should be left as the agent, because that is the way to facilitate learning. However, if the short-term gains (for the patient) of a more active intervention by the therapist clearly outweigh the long-term loss in learning then the therapist should do whatever seems to be required.

\section{Adaptations according to context}

Sometimes the context in which DBT is practised may suggest or require adaptations. These may be the use of special or additional components for particular patient groups. For example, there have been adaptations for people with a major problem of drug misuse, and this variant has been evaluated in a trial, with positive results (Linehan et al, 1999). Likewise, DBT has been used with people with binge eating disorder (Wiser \& Telch, 1999). In an as yet unpublished study in Leicester, DBT has been adapted for people suffering from eating disorder and comorbid BPD by the inclusion of an additional skills training module called 'eatingness'.

The standard out-patient form of DBT needs to be substantially changed if DBT is used in radically different settings such as secure forensic units or if it is generalised to inform the style and culture of a psychiatric day hospital. Such applications and adaptations seem to be accepted within the spirit of DBT.

\section{Stages of treatment}

As a treatment for BPD, DBT seems to be especially well focused on managing the more egregious
Box 2 Commitments in dialectical behaviour therapy

Patient agreements

Stay in therapy for the specified time period

Attend scheduled therapy sessions

Work toward reducing suicidal behaviours as a goal of therapy

Work on problems that arise that interfere with the progress of therapy

Participate in skills training for the specified period

Therapist agreements

Make every reasonable effort to conduct competent and effective therapy

Obey standard ethical and professional guidelines

Be available to the patient for weekly therapy sessions and provide needed therapy backup

Maintain confidentiality

Obtain consent when needed

behaviours of the patient, who may be difficult to engage successfully in more conventional therapies. It is for people showing these difficulties that the more characteristic techniques of DBT seem most appropriate. However, strictly speaking, DBT has four stages.

In the first stage, the targets are to reduce lifethreatening behaviours, therapy-interfering behaviours and quality-of-life-interfering behaviours and to increase behavioural skills. Probably, in practice, most DBT is done in relation to this first stage.

Stage two is targeted at improving the experience of the patient who has reached a state of "quiet desperation", having perhaps overcome the more grossly damaging behaviours as a result of stageone DBT. A characteristic focus of this second stage would be on post-traumatic stress responses.

Stage three is supposed to promote a moving on to "ordinary happiness and unhappiness" by dealing with problems of living.

There is even a fourth stage, which aims at an overcoming of a sense of incompleteness and the development of a "capacity for sustained joy".

These later stages are not much discussed and probably little practised. It seems a fair guess that, not uncommonly, patients move on from DBT to other forms of talking therapy for which the DBT has rendered them more amenable. On these issues the literature is largely silent. 
Box 3 Training for dialectical behaviour therapy

Worldwide

Training is mainly organised through the Behavioral Technology Transfer Group of Seattle (http:/ / www.dbt-seattle.com)

In the UK

A good initial contact would be Susan B. Alexander, Psychological Therapies Research Centre, School of Psychology, University of Leeds, 17 Blenheim Terrace, Leeds LS2 9JT, UK

\section{Training}

Dialectical behaviour therapy is essentially a team treatment and anyone wishing to undergo training in DBT must do so as part of a team. The training assumes that those being trained are experienced clinicians from one of the mental health professions. Acquaintance with CBT may be an advantage but therapists from a wide variety of backgrounds seem to take readily to DBT. Although there are 1- or 2day introductory courses, real training consists of a so-called intensive. An intensive comprises 2 separate weeks of training held a number of months apart. There is an expectation that the team will begin to meet as a weekly consultation group between the 2 weeks and will start their treatment programme. The second of the 2 weeks builds on this experience.

Intensive training courses are held regularly in the USA, and formal training in DBT is controlled by the central organisation in Seattle. So far, two open intensive training programmes have been held in the UK (see Box 3 for sources of information about training). In some wider applications, clinicians may come to work within a DBT ethos without having been formally trained in the treatment.

\section{Conclusions}

Dialectical behaviour therapy is an elaborate and expensive treatment. Furthermore, it is demanding of those who would practice it. However, it has some claims to be an effective treatment for a group of patients who suffer and are at high risk. Other treatments for BPD have less evidence to support any claims for efficacy. Furthermore, people with BPD are often served poorly by less specific treatments. Even with its evident costs, DBT is well worthy of consideration as a response to those patients for whom more routine management seems to be failing. Too often such routine treatment runs the risk of being just as expensive but ineffective or harmful. For the harassed clinican, DBT seems to be well worth a try. At the very least, it provides a coherent framework within which difficult-to-help people may be construed and managed. Typically, patients value it. Anecdotally, clinicians too usually feel good about their practice within DBT. These are not unimportant advantages in a field where too often patients feel hard done by and clinicians feel unappreciated and defeated.

In the wider context of service provision, DBT needs further evaluation. In particular, it needs evaluation in the context of the British National Health Service. Its wider adoption might bring increased benefit and even save money. However, in practice, service innovations and evaluations are difficult to make. They seem to cost too much upfront, as it were. The greater benefits and cost savings that they might bring require initial investment of both effort and money to fund the programmes and their evaluation. There is currently a danger that this promising treatment will be neglected for this kind of reason. There is also another, perhaps less likely, danger that DBT will be widely adopted in the name of evidence-based treatment but in a way that fails to add to the real but modest evidence base on which it now stands.

\section{References}

Aveline, M. (2001) Very brief dynamic psychotherapy. Advances in Psychiatric Treatment, 7, 373-380.

American Psychiatric Association (1994) Diagsnotic and Statistical Manual of Mental Disorders (4th edn) (DSM-IV). Washington, DC: APA.

Denman, C. (2001) Cognitive-analytic therapy. Advances in Psychiatric treatment, 7, 243-252.

Kisely, S. (1999) Psychotherapy for severe personality disorder: exploring the limits of evidence based purchasing. $B M J, 318,1410-1412$.

Linehan, M. M. (1993a) Cognitive-Behavioral Treatment of Borderline Personality Disorder. New York: Guilford Press.

- $(1993 b)$ Skills Training Manual for Treating Borderline Personality Disorder. New York: Guilford Press.

- Armstrong, H. E., Suarez, A., et al (1991) Cognitivebehavioral treatment of chronically parasuicidal borderline patients. Archives of General Psychiatry, 48, 1060-1064.

- Heard, H. L. \& Armstrong, H. E. (1993) Naturalistic follow-up of a behavioral treatment for chronically parasuicidal borderline patients. Archives of General Psychiatry, 50, 971-974

- Tutek, D. A., Heard, H. L., et al (1994) Cognitivebehavioral treatment for chronically parasuicidal borderline patients: interpersonal outcomes. American Journal of Psychiatry, 151, 1771-1776.

-, Schmidt, H., Dimeff, L. A., et al (1999) Dialectical behavior therapy for patients with borderline personality disorder and drug-dependence. American Journal on Addictions, 8, 279-292. 
Mynors-Wallis, L. (2001) Problem-solving treatment in general psychiatric practice. Advances in Psychiatric Treatment, 7 417-425.

Palmer, R. L. (2000) Helping People with Eating Disorders. Chichester: John Wiley \& Sons.

Perry, J. C., Banon, E. \& Ianni, F. (1999) Effectiveness of psychotherapy for personality disorders. American Journal of Psychiatry, 156, 1312-1321.

Wiser, S. \& Telch, C. F. (1999) Dialectical behavior therapy for binge-eating disorder. Journal of Clinical Psychology, 55, $755-768$

\section{Multiple choice questions}

1. The following are usual components of standard DBT:
a weekly skills training group
$\mathrm{b}$ admission to hospital
c keeping a behavioural diary
d availability of 24-hour telephone contact
e willingness to embrace Buddhist beliefs.

2. DBT has been shown to be better than the following in randomised controlled trials:

a CBT in women with BPD and alcohol dependence

b community treatment as usual in men with recurrent self-harm

c CBT in complicated bulimia nervosa

d community treatment as usual in women with recurrent self-harm

e dynamic therapy in BPD.

3. The following techniques are frequently used in DBT:

a interpretation of the transference

b twice-weekly individual therapy sessions

c irreverent communication style

d refusal under almost all circumstances to talk to other professionals involved in the care of the patient

e mindfulness exercises.
4. Skills training groups in DBT have the following characteristics:

a long periods of silence

b attention to group process

c homework exercises

d use of handouts

e attention to individual patient's problems.

5. Full training in DBT should involve:
a training as part of a team
$\mathrm{b}$ being in personal therapy
c a 2-day course
d two 1-week intensive courses
e going to Seattle.

MCQ answers

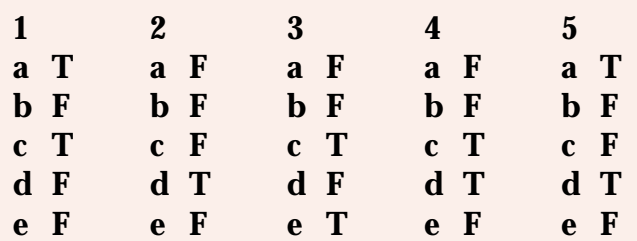

